\title{
General Psychiatry Stress and sleep: a survey based on wearable sleep trackers among medical and nursing staff in Wuhan during the COVID-19 pandemic
}

\author{
Kaiming Zhuo, ${ }^{1,2}$ Cunyou Gao, ${ }^{2,3}$ Xiaohui Wang, ${ }^{2,4}$ Chen Zhang, ${ }^{1,2}$ Zhen Wang (D) ${ }^{1,2}$
}

To cite: Zhuo K, Gao C, Wang $X$, et al. Stress and sleep: a survey based on wearable sleep trackers among medical and nursing staff in Wuhan during the COVID-19 pandemic. General Psychiatry 2020;33:e100260. doi:10.1136/ gpsych-2020-100260

Received 06 May 2020 Revised 20 May 2020 Accepted 25 May 2020

\section{Check for updates}

C Author(s) (or their employer(s)) 2020. Re-use permitted under CC BY-NC. No commercial re-use. See rights and permissions. Published by BMJ.

${ }^{1}$ Shanghai Mental Health Center, Shanghai Jiao Tong University School of Medicine, Shanghai, China

${ }^{2}$ Shanghai Psychological First Aid Team for Wuhan, Shanghai, China

${ }^{3}$ Shanghai Jiading Mental Health Center, Shanghai, China

${ }^{4}$ Shanghai Qingpu Mental Health Center, Shanghai, China

Correspondence to

Dr Zhen Wang;

wangzhen@smhc.org.cn

\section{ABSTRACT}

Backgroud COVID-19 pandemic has significantly affected the sleep health of local medical and nursing staff.

Aim We used wearable pulse oximeters to monitor and screen the medical and nursing staff working in hospitals designated for COVID-19 in the Wuhan area. This study aimed to establish a reliable basis to provide sleep intervention for the medical and nursing staff. Methods Thirty medical and nursing staff members with symptoms of insomnia were instructed to wear medical ring-shaped pulse oximeters to monitor their sleep overnight. We also used the Insomnia Severity Index (ISI) and the Chinese version of the Self-Reporting Questionnaire (SRQ-20) to evaluate the severity of insomnia and mental health status, respectively, for each participant.

Results Among the 30 participants, only 26 completed the screening. Ten cases (38.5\%) demonstrated moderate to severe sleep apnoea-hypopnea syndrome (SAHS) when using an oxygen desaturation index $\geq 15$ times/hour as the cut-off value. Participants with comorbid moderate to severe SAHS had significantly higher ISI and SRQ scores ( $p$ values 0.034 and 0.016 , respectively) than those in the insomnia group. Correlation analysis revealed that ISI was positively correlated with total sleep time (TST) $(r=0.435$, $\mathrm{p}=0.026$ ), and negatively correlated with deep sleep $(r=-0.495, p=0.010)$; furthermore, patient SRQ scores were positively correlated with TST, sleep efficiency (SE) and REM (rapid eyes movement) sleep \% ( $r=0.454$ and $0.389,0.512 ; p=0.020,0.050$ and 0.008 , respectively). Stepwise logistic regression indicated that SRQ-20 and sex were risk factors for insomnia with comorbid SAHS, and their OR values were 1.516 and 11.56 (95\% $\mathrm{Cl} 1.053$ to 2.180 and 1.037 to 128.9 ), respectively.

Conclusion Medical and nursing staff with insomnia showed clear signs of comorbid sleep apnoea attributable to stress. The wearable pulse oximeters accurately monitored the participants' breathing when asleep.

Since December 2019, there have been several cases of an acute respiratory disease occurring in Wuhan City, Hubei Province in China. This disease, which was originally known as novel coronavirus pneumonia and later as the COVID-19, ${ }^{1-3}$ has spread rapidly from Wuhan to the rest of the world. Currently, more than 1 million cases of COVID-19 have been confirmed globally, and the WHO has declared the COVID-19 outbreak a global pandemic. ${ }^{4}$ This pandemic has significantly affected the mental health of local medical and nursing staff. Kang et a $\tilde{p}$ reported that among the mental health of 994 medical and nursing staff working in Wuhan during the epidemic, $22.4 \%$ demonstrated moderate to high levels of depression, anxiety and insomnia, with $6.2 \%$ being diagnosed with severe levels of the same.

Sleep is an indispensable physiological process in maintaining physical health. When facing stressful situations, individuals will need to respond to drastic changes in the external environment, which may cause symptoms such as sleep suppression and increased wakefulness, thus increasing the occurrence of insomnia (including difficulty falling asleep, difficulty maintaining sleep and waking up early), daytime sleepiness, nightmares and daytime dysfunction, among other sleep-related disorders. ${ }^{6}$ Sleep apnoeahypopnea syndrome (SAHS) is a common sleep disorder that primarily manifests in the form of obstructive sleep apnoea-hypopnea syndrome (OSAHS). Approximately $14 \%$ of men and $5 \%$ of women suffer from OSAHS. ${ }^{7}$ Although OSAHS clinically presents with sleepiness and is considerably different from insomnia, an increasing number of studies have shown that OSAHS often coexists with insomnia. Furthermore, the prevalence of comorbid insomnia in OSAHS patients may be as high as $67.4 \% .{ }^{8}$ Conditions that are traumatic and stressful may exacerbate insomnia resulting in disturbed sleep and increased duration of stage 1 light sleep; consequently, these frequent changes in sleep phases further increase upper airway instability and aggravate OSAHS symptoms. ${ }^{9}$ Comorbidity of OSAHS and insomnia in stressful conditions 
may further amplify physical harm by increasing the risk of cardiovascular and cerebrovascular diseases. ${ }^{1011}$ Therefore, it is imperative to monitor the medical and nursing staff diagnosed with OSAHS and comorbid insomnia in the current scenario.

Wearable medical pulse oximeters were administered to monitor and screen the medical and nursing staff working in COVID-19 designated hospitals in the Wuhan area and evaluate their mental health to elucidate the psychological and physiological effects of stress on sleep and breathing. We hope that our findings will provide a reliable basis to administer sleep intervention to the pertinent medical and nursing staff members.

\section{DATA AND METHODS}

This study was implemented in March 2020.

\section{Participants}

We included medical and nursing staff members working in Wuhan Children's Hospital, Wuhan Central Hospital and other COVID-19 designated hospitals between January and March 2020. Prior to their enrolment, the participants were clinically evaluated by psychiatrists in the Shanghai Psychological First Aid Team for Wuhan.

\section{Inclusion criteria}

Patients (1) aged $\geq 18$ years; (2) frequently presenting with symptoms of nocturnal insomnia, such as difficulty in falling asleep, difficulty in maintaining sleep and waking up early, which were accompanied by daytime fatigue, decline in attention or memory, impulsiveness and irritability, sleepiness and other symptoms since they began participating in COVID-19 efforts, as well as meeting the ICSD-3 (International Classification of Sleep Disorders-3) diagnostic criteria for short-term insomnia; (3) who have not taken benzodiazepine or non-benzodiazepine sedative hypnotics within the past month; and (4) who have provided a written informed consent.

\section{Exclusion criteria}

(1) Patients with concomitant cardiovascular, lung, liver, kidney, hematopoietic and other severe primary diseases and those with severe organic brain disease and mental illnesses; (2) patients with a history of alcoholism or use of other psychoactive substances; and (3) pregnant or breastfeeding women.

Medical ring-shaped pulse oximeter is a new type of wearable preliminary sleep screening instrument that can monitor pulse rate and blood oxygen changes overnight. Pulse rate indicators include mean pulse rate, minimum/ maximum pulse rate and nocturnal pulse elevation index (ie, the number of sleeping heart rate fluctuations $>6$ times/min). Blood oxygen indicators include oxygen desaturation index $\left(\mathrm{ODI}_{4}\right.$; ie, the number of times blood oxygen saturation decreases by $\geq 4 \%$ per hour), mean percutaneous oxygen saturation $\left(\mathrm{MSpO}_{2}\right)$, percentage of total sleeping time spent with blood oxygen saturation
$<90 \%$ (TS 90\%), among others. Studies conducted in China and globally have shown that $\mathrm{ODI}_{4}$ is positively correlated with the Apnoea-Hypopnea Index, and an $\mathrm{ODI}_{4} \geq 15$ times/hour was considered to provide an adequate degree of sensitivity and specificity to screen moderate to severe OSAHS. ${ }^{12}$

The Insomnia Severity Index (ISI) is an instrument developed by Morin et al to measure the self-perceived symptoms of insomnia in the prior 2 weeks. ${ }^{13}$ Compared with the Pittsburgh Sleep Quality Index and Epworth Sleepiness Scale, the ISI specifically assesses the severity of insomnia. ISI includes seven items, is relatively simple and convenient to administer and has a high level of empirical validity. It has been widely applied in clinical research and has demonstrated an excellent degree of consistency and effectiveness in reflecting the quality of sleep.

The Self-Reporting Questionnaire (SRQ-20) is an questionnaire developed by the WHO to screen for mental disorders and was designed specifically for developing countries. It consists of 20 questions, each of which may be scored as 0 or 1 , and a higher score indicates a higher prominence of symptoms associated with mental disorders. Previously, this questionnaire was applied in the postdisaster mental health survey of the population, and it demonstrated considerably consistency reliability and criterion-related validity. ${ }^{14}$

\section{Statistical analysis}

Statistically analysis was performed using SPSS V.19.0. Measurement data obtained by monitoring were expressed as $\chi(-) \pm s$, and count data as percentage (\%). T-tests and non-parametric Wilcoxon tests were respectively performed based on whether the data were normally distributed or not; $\chi^{2}$ tests were performed to compare the incidences between two groups. We used the Pearson and Spearman correlation to analyse normally distributed and non-normally distributed measurement data, respectively. Stepwise logistic regression was performed to analyse the independent risk factors of insomnia with comorbid OSAHS. $\mathrm{P}<0.05$ indicated that the difference was statistically significant.

\section{RESULTS}

\section{General demographics and questionnaire survey}

Forty-nine medical and nursing staff members were screened of which 30 enrolled. Finally 26 participants (14 men and 12 women) completed the study. The participant demographics, along with the questionnaire results are presented in table 1 . The monitoring results of medical pulse oximetry are shown in table 2 .

Analysis of ring-shaped pulse oximetric monitoring indicators The total sleep time (TST), sleep efficiency (SE), light sleep stage, deep sleep stage, REM sleep stage, ODI, frequency of oxygen desaturation, $\mathrm{TS} 90 \%, \mathrm{MSpO}_{2}$ and mean pulse rate were monitored using the pulse oximeter, and their results are shown in table 2. Using ODI $\geq 5$ 


\begin{tabular}{lc}
\hline Table 1 & General demographics and questionnaire results \\
\hline Item & Mean (SD) (n=26) \\
\hline Age (year) & $41.92(9.32)$ \\
Sex (male/female, case) & $14 / 12$ \\
BMI $\left(\mathrm{kg} / \mathrm{m}^{2}\right)$ & $23.41(2.86)$ \\
ISI & $7.69(5.11)$ \\
SRQ-20 & $4.19(3.47)$
\end{tabular}

BMI, body mass index; ISI, Insomnia Severity Index; SRQ-20, SelfReporting Questionnaire.

times/hour as the cut-off value for classification revealed that 14 cases $(53.8 \%)$ presented with SAHS. Considering the fact that an ODI $\geq 15$ times/hour was used to classify moderate to severe disorder, 10 cases $(38.5 \%)$ were found to have moderate to severe SAHS.

Using the cut-off value of ODI $\geq 15$ times/hour, we categorised the participants with an ODI $<15$ times/hour into the insomnia group, while those with $\geq 15$ times/hour to the comorbid moderate to severe SAHS group, following which an intergroup comparison was performed. We used the non-parametric Wilcoxon test since both sets of data were not normally distributed. The results of the same are presented in table 3 .

Given the significant intergroup differences with regard to the number of male and female participants, the analysis of covariance was performed with the patient sex as a covariate. The results indicated that the intergroup differences for ISI, SRQ, ODI, TS90\% and $\mathrm{MSpO}_{2}$ remained statistically significant $(\mathrm{F}=7.738,7.472,114.9$, 6.925 and $11.64, \mathrm{p}=0.011,0.012,0.000,0.015$ and 0.002 , respectively).

Spearman correlation analysis for age, sex, ISI and SRQ with the various sleep indicators revealed that ISI was positively correlated with SRQ and TST $(r=0.685,0.435$; $\mathrm{p}<0.001$ and $\mathrm{p}<0.026$ ) and negatively correlated with deep sleep $(r=-0.495, \mathrm{p}=0.010)$; furthermore, $\mathrm{SRQ}$ was

\begin{tabular}{lc}
\hline Table 2 Results of pulse oximetry & \\
\hline Item & Mean (SD) (n=26) \\
\hline Total sleep time (min) & $442.0(55.0)$ \\
Sleep efficiency (\%) & $86.37(4.93)$ \\
\hline Light sleep stage (\%) & $46.63(8.06)$ \\
Deep sleep stage (\%) & $20.88(6.09)$ \\
REM sleep stage (\%) & $19.18(4.13)$ \\
ODI & $16.16(17.49)$ \\
\hline Frequency of oxygen desaturation & $123.92(136.58)$ \\
\hline TS90\% & $4.61(10.22)$ \\
$\mathrm{MSpO}_{2}$ & $62.00(7.08)$ \\
$\mathrm{Mean} \mathrm{pulse} \mathrm{rate}$ & $62.00(7.08)$ \\
\hline
\end{tabular}

$\mathrm{MSpO}_{2}$, mean percutaneous oxygen saturation; ODI, oxygen desaturation index; TS90\%, percentage of time spent at $\mathrm{SpO}_{2}$ below $90 \%$. positively correlated with TST, SE and REM\% ( $r=0.454$, $0.389,0.512 ; \mathrm{p}=0.020,0.050$ and 0.008 , respectively).

\section{Stepwise logistic regression}

Stepwise multiple logistic regression was performed using the presence of SAHS as the dependent variable, along with other variables such as age, sex, BMI, ISI score, SRQ-20 score and sleep quality (see table 4 ). The outcome of the $\chi^{2}$ test performed after including SRQ-20 and sex in the regression equation was as follows: $\chi(-)$ ${ }^{2}=11.84, \mathrm{p}=0.003$; considering which, the regression equation was statistically significant. Therefore, the SRQ-20 score and patient sex were considered to be risk factors for insomnia with comorbid SAHS, and their OR values were 1.516 and 11.56 (95\% CI 1.053 to 2.180 and 1.037 to 128.9), respectively.

\section{DISCUSSION}

\section{Main findings}

Outcomes of the screening conducted in this study revealed that the incidence of comorbid moderate to severe SAHS in insomnia patients was $38.5 \%(10 / 26)$. However, using a cut-off value of ODI $\geq 5$ times/hour demonstrated that the proportion of SAHS patients with comorbid insomnia was even higher $(53.8 \%)$. This finding further validates the outcomes of previous investigations using polysomnography. Guilleminault $e a^{15}$ first proposed the concept of OSAHS comorbid with insomnia in 1973, while Krakow et $\mathrm{al}^{16}$ reported that OSAHS comorbidity was clinically significant in more than $50 \%$ of insomnia patients. The high comorbidity rate of OSAHS and insomnia suggests that these two diseases may not be purely concomitant but may share common pathophysiological mechanisms. In addition, the overactivation of the hypothalamic-pituitary-adrenal (HPA) axis under stress may be the link between the two.

In cases of traumatic stress, acute stress suppresses REM sleep and increases the duration of non-REM sleep, which is followed by restorative sleep compensation, increased sleep time and increased REM sleep. ${ }^{17}$ However, persistent or repeated chronic stress exposure may have a more negative impact on sleep, resulting in the presentation of sleep disorders, insomnia and other problems. ${ }^{18}$ In the presence of an active HPA axis, individuals may experience a lighter sleep, increasing awakenings and sleep fragmentation. ${ }^{19}$ Fragmented sleep can subsequently excite the sympathetic catecholamine system and elevate cortisol levels, which eventually results in persistent insomnia. ${ }^{20}$ Repeated awakenings and an increased frequency of light sleep can also lead to frequent sleep phase changes and upper airway instability, thereby exacerbating SAHS symptoms. ${ }^{21}$ SAHS-induced decrease in blood oxygen saturation generates hypoxic stress, which further activates the HPA axis while increasing the patients' breathing rate to maintain adequate alveolar ventilation. This results in repeated microarousals during 
Table 3 Intergroup comparison of characteristics and sleep parameters of participants

\begin{tabular}{|c|c|c|c|c|}
\hline Group & $\begin{array}{l}\text { Insomnia group } \\
(n=16)\end{array}$ & $\begin{array}{l}\text { Comorbid SAHS group } \\
(n=10)\end{array}$ & $\begin{array}{l}\mathbf{Z} / \chi(-)^{2} \\
\text { Value }\end{array}$ & $P$ value \\
\hline Age & 41.68 (10.33) & $42.30(7.95)$ & 0.106 & 0.916 \\
\hline BMI & $22.77(2.61)$ & 24.42 (3.09) & 1.476 & 0.140 \\
\hline ISI & $5.87(4.28)$ & $10.60(5.16)$ & 2.115 & $0.034^{*}$ \\
\hline TST (min) & $432.31(59.34)$ & $457.60(45.85)$ & 1.081 & 0.280 \\
\hline SE (\%) & $85.77(4.48)$ & 87.33 (5.69) & 0.659 & 0.510 \\
\hline LS (\%) & $46.33(9.17)$ & $47.11(6.31)$ & 0.211 & 0.833 \\
\hline DS (\%) & $21.50(5.81)$ & $19.90(6.70)$ & 1.107 & 0.268 \\
\hline REM (\%) & $17.94(2.45)$ & $21.19(5.50)$ & 1.450 & 0.147 \\
\hline TS90\% & $0.51(1.63)$ & $11.17(14.42)$ & 3.393 & $\mathrm{p}<0.001^{*}$ \\
\hline $\mathrm{MSpO}_{2}$ & $97.28(1.59)$ & $94.43(2.04)$ & 3.374 & $0.001^{*}$ \\
\hline Pulse rate, $\mathrm{P}$ & $59.87(7.31)$ & $65.40(5.42)$ & 2.218 & $0.027^{*}$ \\
\hline
\end{tabular}

${ }^{*} \mathrm{p}<0.05$

BMI, body mass index; DS, deep sleep stage; ISI, Insomnia Severity Index; LS, light sleep stage; $\mathrm{MSpO}_{2}$, mean percutaneous oxygen saturation; ODI, oxygen desaturation index; SE, sleep efficiency; SRQ, self-reporting questionnaire; TS90\%, percentage of time spent at SpO2 below $90 \%$; TST, total sleep time.

sleep, which disrupts the continuity and periodicity of sleep, which further fragments the sleep cycle. ${ }^{22}$

The Insomnia Severity Index (ISI) has demonstrated an acceptable degree of consistency and validity in reflecting the severity of insomnia. ${ }^{13}$ Our screening results showed that participants with insomnia and comorbid SAHS scored higher on the ISI than those with insomnia alone, and ISI was negatively correlated with the duration of deep sleep. These findings indicated that the lack of deep sleep continues to be the main cause of insomnia and that SAHS is a key factor responsible for the fragmentation and superficialisation of the sleep process. ${ }^{22}$ ISI was positively correlated with TST $(\mathrm{r}=0.435, \mathrm{p}=0.026)$, which is inconsistent with the outcomes of previous studies that have reported a decrease in subjective and objective TST in insomnia patients. This may be attributed to the concomitant presentation of insomnia and SAHS that may further damage the sleep structure; alternatively, it may be due to the relatively small sample size of this study.

The self-reporting questionnaire (SRQ-20) has been translated into more than 10 languages for use in the corresponding regions around the world, and each version has been scrutinised for its reliability and validity. ${ }^{142324}$ Jiang et $\mathrm{al}^{14}$ studied the reliability and validity of the Chinese version of the SRQ-20, which was administered 3 weeks after the Wenchuan earthquake, and reported that this questionnaire had an acceptable degree of consistency reliability and criterion-related validity in reflecting the postdisaster traumatic stress experienced by victims. In this study, participants with insomnia and comorbid SAHS showed significantly higher SRQ scores than those with insomnia alone. The results of logistic regression analysis also indicated that the SRQ-20 score was a risk factor for insomnia with comorbid SAHS, with a higher score indicating a greater risk for insomnia with comorbid SAHS. This finding suggested that SAHS comorbidity may further introduce psychological problems in individuals who had previously experienced traumatic stress. Some studies have shown that OSAHS patients often concomitantly present with depression and anxiety, which are the factors that significantly contribute to a decline in their quality of life and lead to incidence rates of $34 \%-56 \%{ }^{25}$

Table 4 Logistic regression prediction model parameters for sleep perception disorders

\begin{tabular}{lllllll}
\hline & \multicolumn{2}{l}{ Regression coefficient } & & & & \\
Item & $\boldsymbol{\beta}$ & SE & Wald & OR & $\mathbf{9 5 \%}$ Cl & P value \\
\hline Sex & 2.448 & 1.230 & 3.957 & 11.56 & 1.037 to 128.9 & 0.047 \\
SRQ-20 & 0.416 & 0.186 & 5.019 & 1.516 & 1.053 to 2.180 & 0.025
\end{tabular}

$R^{2}=0.497$.

SRQ-20, Self-Reporting Questionnaire. 
Our correlation analyses revealed that the participants' SRQ-20 scores were positively correlated with TST, SE and REM\%, suggesting the significant impact of psychological factors on the participants' sleep duration, quality and structure. Given that the participants had been under stress for 2 months when this screening was performed, they had been exposed to its effects for a relatively long period of time, which suggested the possibility of chronic stress exposure. Therefore, individuals may exhibit restorative sleep compensation, along with increased sleep duration and REM sleep, which is consistent with the outcomes of previous investigations. ${ }^{17}$

Middle-aged and overweight men are reportedly considered to be at risk for OSAHS. ${ }^{26}$ We also observed that the proportion of men was higher in the comorbid SAHS group, while the results of logistic regression analysis showed that men were more likely to have insomnia with comorbid SAHS. However, when considering sex as a covariate for further analysis, the results showed that the intergroup differences in ISI, SRQ, ODI, TS90\% and $\mathrm{MSpO}_{2}$ persisted, which suggests that sex may generate comorbid OSAHS in men with insomnia. However, the severity of SAHS and the degree of psychological distress were not affected by sex differences.

Wearable devices significantly improved the convenience of using sleep trackers, especially when we could continue to monitor the patients at their residence, which ensured that the monitoring environment was closer to natural sleep. However, research in China and globally on wearable sleep trackers is relatively limited. A study has shown that ${ }^{27}$ compared with portable EEG, wearable sleep trackers had better sensitivity $(92.1 \%)$ and accuracy $(88.5 \%)$ when evaluating TST and SE but showed significant bias when determining sleep latency and sleep cycle. Hence, this approach has its limitations.

\section{Limitations}

This study has certain limitations. First, this was a crosssectional observational study. Although wearable pulse oximeters can easily monitor participants' breathing during sleep, its clinical applications are considerably limited, and further testing is required to verify its reliability. Second, mental health screening was only performed using the ISI and SRQ and a comprehensive psychological evaluation was not conducted on the participants' stress severity, anxiety, depression and other psychological conditions. Lastly, the small sample size of this study may have affected our screening results. Therefore, future studies should effectively record clinical symptoms and signs, improve the parameters for psychological and sleep questionnaires and confirm the diagnosis of suspected insomnia with comorbid SAHS using overnight polysomnography, thereby exploring the inherent connections of stress, insomnia and SAHS to a greater degree.

\section{Implications}

During crisis intervention, sedative-hypnotic drugs, such as benzodiazepines, have been widely used to alleviate symptoms such as anxiety, tension and insomnia after stress and can rapidly improve clinical symptoms. Nevertheless, insomnia with comorbid SAHS has long been neglected in previous reports, and the use of benzodiazepines may worsen SAHS symptoms. Therefore, when assessing the situation during future emergency rescues, it is necessary to combine symptoms, signs and other clinical data to thoroughly screen insomnia patients with comorbid SAHS and avoid the adverse reactions caused by benzodiazepines.

Acknowledgements The authors would like to acknowledge those subjects taking part in this study and the support of the funding sources.

Contributors KZ participated in the design and data collection for the study and drafted the first version of the manuscript. CG and XW enrolled and clinically evaluated study subjects. CZ carried out the clinical diagnosis and critically reviewed the manuscript. ZW was responsible for drafting and revising the main contents of the paper. All authors read and approved the final manuscript.

Funding This work was supported by the grant from Shanghai Jiao Tong University (YG2020YQ25 and YG2017MS43) and Young Doctor Training Program of Shanghai Municipal Health Commission (2014ZYJB0002).

Competing interests None declared.

Patient consent for publication Not required.

Ethics approval The research protocol was approved by Institutional Review Board of Shanghai Mental Health Center (2020-10). This study was conducted in accordance with the 1964 Declaration of Helsinki and its later amendments or comparable ethical standards. The subjects were assured of the following: their participation was voluntary, they could withdraw at any time without facing any negative consequences, their anonymity would be protected and the data obtained would not be used for purposes other than the present research. All participants provided their written informed consent. The research purpose and guarantee to protect the privacy of the subjects were explained verbally as well as in written form to the participants by the doctor.

Provenance and peer review Not commissioned; externally peer reviewed.

Data availability statement Data are available on reasonable request. The raw data required to reproduce these findings cannot be shared at this time as the data also forms part of an ongoing study.

Open access This is an open access article distributed in accordance with the Creative Commons Attribution Non Commercial (CC BY-NC 4.0) license, which permits others to distribute, remix, adapt, build upon this work non-commercially, and license their derivative works on different terms, provided the original work is properly cited, appropriate credit is given, any changes made indicated, and the use is non-commercial. See: http://creativecommons.org/licenses/by-nc/4.0/.

ORCID iD

Zhen Wang http://orcid.org/0000-0003-4319-5314

\section{REFERENCES}

1 Lu H, Stratton CW, Tang Y-W. Outbreak of pneumonia of unknown etiology in Wuhan, China: the mystery and the miracle. J Med Virol 2020;92:401-2.

2 Hui DS, I Azhar E, Madani TA, et al. The continuing 2019-nCoV epidemic threat of novel coronaviruses to global health - The latest 2019 novel coronavirus outbreak in Wuhan, China. Int $J$ Infect Dis 2020;91:264-6.

3 Paules $\mathrm{Cl}$, Marston HD, Fauci AS. Coronavirus Infections-More than just the common cold. JAMA 2020;323:707.

4 WHO. Coronavirus disease (COVID-19) outbreak situation [M] 2020.

5 Kang L, Ma S, Chen M, et al. Impact on mental health and perceptions of psychological care among medical and nursing staff in Wuhan during the 2019 novel coronavirus disease outbreak: a cross-sectional study. Brain Behav Immun 2020. doi:10.1016/j. bbi.2020.03.028. [Epub ahead of print: 30 Mar 2020].

6 Otsuka Y, Kaneita Y, Itani O, et al. Relationship between stress coping and sleep disorders among the general Japanese population: a nationwide representative survey. Sleep Med 2017;37:38-45. 
7 Peppard PE, Young T, Barnet JH, et al. Increased prevalence of sleep-disordered breathing in adults. Am J Epidemiol 2013;177:1006-14

8 Lichstein KL, Justin Thomas S, Woosley JA, et al. Co-Occurring insomnia and obstructive sleep apnea. Sleep Med 2013;14:824-9.

9 Krakow B, Lowry C, Germain A, et al. A retrospective study on improvements in nightmares and post-traumatic stress disorder following treatment for co-morbid sleep-disordered breathing. $J$ Psychosom Res 2000;49:291-8.

10 Vargas I, Vgontzas AN, Abelson JL, et al. Altered ultradian cortisol rhythmicity as a potential neurobiologic substrate for chronic insomnia. Sleep Med Rev 2018;41:234-43.

11 Drager LF, McEvoy RD, Barbe F, et al. Sleep apnea and cardiovascular disease: lessons from recent trials and need for team science. Circulation 2017:136:1840-50.

12 Vázquez JC, Tsai WH, Flemons WW, et al. Automated analysis of digital oximetry in the diagnosis of obstructive sleep apnoea. Thorax 2000;55:302-7.

13 Bastien $\mathrm{CH}$, Vallières $\mathrm{A}$, Morin $\mathrm{CM}$. Validation of the insomnia severity index as an outcome measure for insomnia research. Sleep Med 2001;2:297-307.

14 Jiang $X$, Wang Z, Wang $X$, et al. Reliability and validity of the Self-Reporting Questionnaire (SRQ-20) in high school students in Wenchuan earthquake area [J]. Chinese Mental Health Journal 2010;24:313-7.

15 Guilleminault C, Eldridge FL, Dement WC. Insomnia with sleep apnea: a new syndrome. Science 1973;181:856-8.

16 Krakow B, Melendrez D, Ferreira E, et al. Prevalence of insomnia symptoms in patients with sleep-disordered breathing. Chest 2001;120:1923-9.

17 Sanford LD, Suchecki D, Meerlo P. Stress, arousal, and sleep. Curr Top Behav Neurosci 2015;25:379-410.
18 Neckelmann D, Mykletun A, Dahl AA. Chronic insomnia as a risk factor for developing anxiety and depression. Sleep 2007;30:873-80.

19 Buckley TM, Schatzberg AF. On the interactions of the hypothalamicpituitary-adrenal (HPA) axis and sleep: normal HPA axis activity and circadian rhythm, exemplary sleep disorders. J Clin Endocrinol Metab 2005;90:3106-14.

20 Balbo M, Leproult R, Van Cauter E. Impact of sleep and its disturbances on hypothalamo-pituitary-adrenal axis activity. Int $\mathrm{J}$ Endocrinol 2010;2010:1-16.

21 Sériès F, Roy N, Marc I. Effects of sleep deprivation and sleep fragmentation on upper airway collapsibility in normal subjects. Am J Respir Crit Care Med 1994:150:481-5.

22 Svanborg E, Larsson $\mathrm{H}$. Development of nocturnal respiratory disturbance in untreated patients with obstructive sleep apnea syndrome. Chest 1993;104:340-3.

23 Al-Subaie AS, Mohammed K, Al-Malik T. The Arabic self-reporting questionnaire (SRQ) as a psychiatric screening instrument in medical patients. Ann Saudi Med 1998;18:308-10.

24 Giang KB, Allebeck P, Kullgren G, et al. The Vietnamese version of the self reporting questionnaire 20 (SRQ-20) in detecting mental disorders in rural Vietnam: a validation study. Int J Soc Psychiatry 2006;52:175-84.

25 Saunamäki T, Jehkonen M. Depression and anxiety in obstructive sleep apnea syndrome: a review. Acta Neurol Scand 2007;116:277-88.

26 Punjabi NM, Sorkin JD, Katzel LI, et al. Sleep-Disordered breathing and insulin resistance in middle-aged and overweight men. Am J Respir Crit Care Med 2002;165:677-82.

27 Svensson T, Chung U-I, Tokuno S, et al. A validation study of a consumer wearable sleep tracker compared to a portable EEG system in naturalistic conditions. J Psychosom Res 2019;126:30.

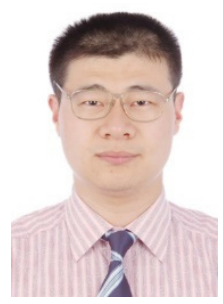

Kaiming Zhuo obtained a master's degree from the Division of Psychosis at the Shanghai Mental Health Center, Shanghai Jiao Tong University School of Medicine in 2014. He has been working at the Shanghai Mental Health Center, Shanghai Jiao Tong University School of Medicine since 2005 where he is an attending psychiatrist. He has been to Inner Mongolia and Wuhan as a member of the national medical team for psychological rescue from 2019 to 2020. His main research interests include early psychosis and sleep. 
Correction: Stress and sleep: a survey based on wearable sleep trackers among medical and nursing staff in Wuhan during the COVID-19 pandemic

Zhuo K, Gao C, Wang X, et al. Stress and sleep: a survey based on wearable sleep trackers among medical and nursing staff in Wuhan during the COVID-19 pandemic. Gen Psychiatr 2020;33:e100260. doi: 10.1136/gpsych-2020-100260.

This article was previously published with an error in the text.

In the 'Statistical analysis' section under 'Data and methods', should read mean (SD) not $\chi(-) \pm s$,

In the 'Stepwise logistic regression' section under 'RESULTS', should read $\chi^{2}$ not $\chi(-)^{2}$.

In table 3 , the fourth column heading should read $\chi^{2}$ not $\chi(-)^{2}$.

\section{(2) \\ OPEN ACCESS}

Open access This is an open access article distributed in accordance with the Creative Commons Attribution Non Commercial (CC BY-NC 4.0) license, which permits others to distribute, remix, adapt, build upon this work non-commercially, and license their derivative works on different terms, provided the original work is properly cited, appropriate credit is given, any changes made indicated, and the use is non-commercial. See: http://creativecommons.org/licenses/by-nc/4.0/.

(c) Author(s) (or their employer(s)) 2020. Re-use permitted under CC BY-NC. No commercial re-use. See rights and permissions. Published by BMJ.

General Psychiatry 2020;33:e100260corr1. doi:10.1136/gpsych-2020-100260corr1

A) Check for updates 